\title{
Poster: Detection of Misplaced Stationary RFID Tags
}

\author{
Jiaqing Luo \\ School of Computer Science and Engineering \\ University of Electronic Science \& Technology of China \\ Chengdu, Sichuan, China \\ csjluo@hotmail.com
}

\begin{abstract}
A smart shelving system can visualize stock data in real time by leveraging item-level RFID tagging. The detection of misplaced tags on stationary shelved items is very challenging due to position ambiguity, phase wrapping, device diversity, and phase ambiguity. We propose an effective way of detecting misplaced tags, called FINDS, that requires neither antenna movement nor external disturbances.
\end{abstract}

\section{CCS CONCEPTS}

- Networks $\rightarrow$ Mobile networks.

\section{KEYWORDS}

RFID; misplaced tags; stationary tags

\section{ACM Reference Format:}

Jiaqing Luo and Kang G. Shin. 2019. Poster: Detection of Misplaced Stationary RFID Tags. In The 17th Annual International Conference on Mobile Systems, Applications, and Services (MobiSys '19), June 17-21, 2019, Seoul, Republic of Korea. ACM, New York, NY, USA, 2 pages. https://doi.org/10.1145/3307334.3328628

\section{MOTIVATION}

Smart shelving replaces visual checks by the store staff, and enables consistent shelf-space allocation compliance by automating shelf-level management of items placement and leveraging item-level tagging to ensure adherence to the supplier's shelf-space allocation.

The main challenge of smart shelving is to locate, in real time, passive tags. Prior work on locating tags - that includes determination of their relative and absolute positions - is either dynamic or static. The former requires to move RFID tags or reader antennas in order to monitor RF signal changes as a time series. The latter requires high-density reference tags or an expensive infrastructure to analyze RF signal differences. Unfortunately, both approaches have limitations in detecting misplaced tags on smart shelves. First, tags usually do not move as items on shelves remain stationary for most of the time. Second, the movement of reader antennas usually needs to be continuous and stable. Third,

Permission to make digital or hard copies of part or all of this work for personal or classroom use is granted without fee provided that copies are not made or distributed for profit or commercial advantage and that copies bear this notice and the full citation on the first page Copyrights for third-party components of this work must be honored. For all other uses, contact the owner/author(s).

MobiSys '19, June 17-21, 2019, Seoul, Republic of Korea

(C) 2019 Copyright held by the owner/author(s).

ACM ISBN 978-1-4503-6661-8/19/06.

https://doi.org/10.1145/3307334.3328628

\author{
Kang G. Shin \\ Electrical Engineering and Computer Science \\ The University of Michigan - Ann Arbor \\ Ann Arbor, Michigan, U.S.A. \\ kgshin@umich.edu
}

the expensive infrastructure is unattractive for large-scale deployment.

To overcome these limitations, we attempt to make the measured phase coincide with the theoretical phase, and identify the phase shifts caused by misplacement of tags. In our preliminary experiments, we were able to achieve $>0.92$ accuracy only with 2 antennas.

\section{CHALLENGES}

We conducted a series of experiments with COTS ImpinJ readers and Alien tags. These experiments were designed to identify the potential difficulties in positioning tags with stationary devices. The system is oriented counter-clockwise with respect to $z$-axis. For simplicity, we only deploy tags in the $x y$-plane.

\subsection{Position Ambiguity}

Tags placed at different positions may yield the same measured phase $\theta$ due to the cycle slip of phase. $\theta$ repeats from 0 to $2 \pi$ every half wavelength $\left(\frac{\lambda}{2} \approx 0.163\right)$. We placed an antenna $A_{1}$ at $(0,0,1.2)$, and two tags $T_{1}$ and $T_{2}$ at $(-0.037,0.306$, $0)$ and $(-0.248,0.681,0)$. The distances from $A_{1}$ to $T_{1}$ and $T_{2}$ are $d_{1}^{1}=\left\|\overrightarrow{A_{1} T_{1}}\right\|=1.239$ and $d_{2}^{1}=\left\|\overrightarrow{A_{1} T_{2}}\right\|=1.402$, respectively. The difference of distance $\Delta d_{2,1}^{1}=d_{2}^{1}-d_{1}^{1}=0.162$ is very close to $\frac{\lambda}{2}$. Assuming that $\theta_{1}^{1}$ and $\theta_{2}^{1}$ are $T_{1}$ 's and $T_{2}$ 's measured phases from $A_{1}$, Fig. 1 shows that $\theta_{1}^{1}$ and $\theta_{2}^{1}$ almost overlap.

\subsection{Phase Wrapping}

Since the theoretical phase $\Theta$ has a normal range $[0,2 \pi)$, the measured phase $\theta=\Theta+\mu$ may exceed the range $[0,2 \pi)$ and contain one or more $2 \pi$ jumps. In such a case, $\theta$ will be wrapped around to stay within the normal range $[0,2 \pi)$. We placed an antenna $A_{1}$ at $(0.6,0.3,1.386)$ and 20 tags in a $4 \times 5$ array. [1] suggests tags to be separated from each other by about $0.1 \mathrm{~m}$. To reduce mutual interferences, we separate two adjacent tags by $0.15 \mathrm{~m}$. Fig. 2 shows that $\theta$ does not vary with $\Theta$. Taking $T_{6}$ and $T_{8}$ as examples, $\theta_{6}^{1}-\Theta_{6}^{1}=$ $4.429-2.045=2.383$, while $\theta_{8}^{1}-\Theta_{8}^{1}=4.786-0.455=4.331$. We calculate the Pearson correlation coefficient for $\theta$ and $\Theta$ to test whether there is a linear correlation between them. The coefficient of correlation is 0.429 , implying that there is a moderate linear correlation between $\theta$ and $\Theta$.

\subsection{Device Diversity}

Device diversity includes diversities of tags and the reader antennas. Prior research has shown device diversity to have 


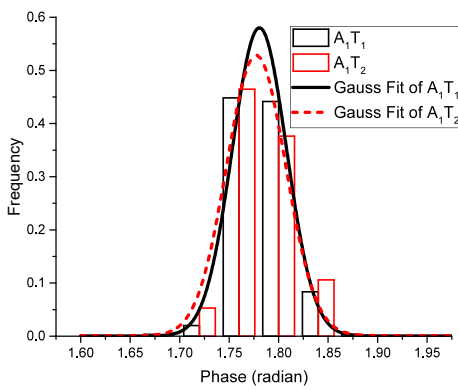

Figure 1: Ambiguity

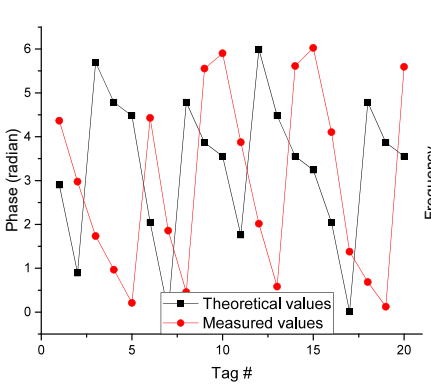

Figure 2: Wrapping

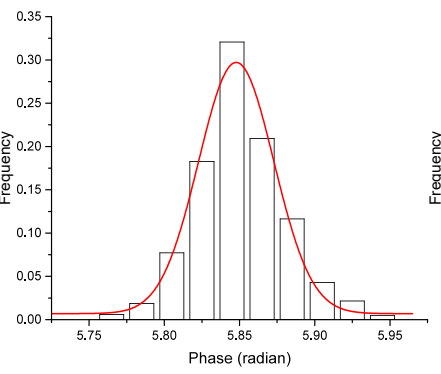

Figure 3: Tags

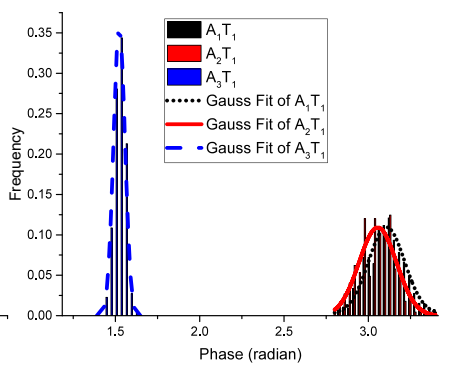

Figure 4: Antennas a great effect on the phase measurement, thus leading to a natural question "can we eliminate this effect by choosing devices of the same model?" We first place an antenna at $(0,0.447,1.2)$ and 60 Alien AZ-9346 tags at $(0,0,0)$ in turn. The measured phase values are long-term averages. Fig. 3 shows that $\theta$ follows a Gaussian distribution with mean 5.85 and standard deviation 0.025 . This effect of the measurement error is negligible. We deployed three Laird S9025PR antennas $A_{1}, A_{2}$ and $A_{3}$ at $(-0.3,0.073,1.2)$ in turn, and utilized them to interrogate tag $T_{1}$ placed at $(0,0$, $0)$. These antennas are of the same model but from different batches. $A_{1}$ and $A_{2}$ 's batch numbers are 1316 while $A_{3}$ 's batch number is 1245 . Fig. 4 shows $\theta_{1}^{1}$ very close to $\theta_{1}^{2}$, while $\theta_{1}^{3}$ is very different from the other two.

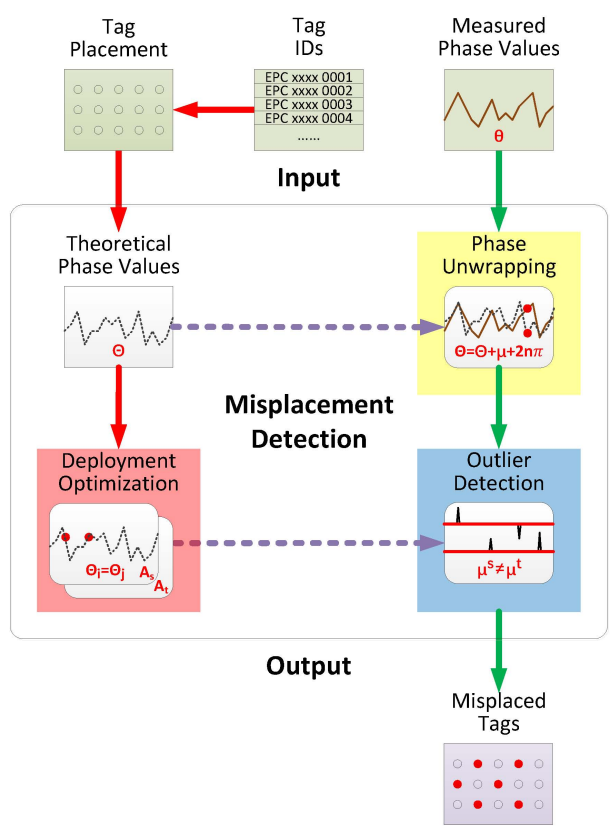

Figure 5: Basic FINDS framework

\section{MISPLACEMENT DETECTION}

The misplacement detection can be viewed as a special type of static positioning. We assume that tags are placed at a set of fixed positions. This is a reasonable assumption for clothing or unmanned shelves. Our basic idea is to calibrate the measured phase values and detect the abnormal phase shifts caused by misplaced tags. FINDS neither collects signal fingerprints (RSS or phase) a priori nor tracks signal changes to support a cold start.

As shown in Fig. 5, FINDS consists of input, misplacement detection and output. The input includes tag IDs, phase measurements, and tag placement. The misplacement is composed of deployment optimization, phase unwrapping, and outlier detection. The output is the tag IDs and the positions of misplaced tags.

Specifically, FINDS takes several steps to detect misplaced tags. First, it optimizes the deployment of antennas to reduce position ambiguities. It later collects tag IDs and phase measurements by interrogating tags. It then obtains the expected positions of tags from the pre-determined tag placement, followed by calculation of the theoretical phase values corresponding to the expected positions. Next, it detects and corrects phase wraps according to a simple yet effective criterion, comparing the measured and the theoretical phase values. Finally, it eliminates the effect of systemic errors and detects the anomalous phase shifts caused by misplaced tags.

Some basic principles and algorithms of FINDS can also be used in other applications. For instance, we can deploy some reference tags at known positions, and then estimate systemic errors by the unwrapping, which could be helpful for positioning or tracking tags at unknown positions.

\section{ACKNOWLEDGMENTS}

Jiaqing Luo was supported in part by the National Natural Science Foundation of China under Grant 61602093, the Key Project of Science and Technology of Sichuan under Grants 2018FZ0099 and 18ZDYF2554 and the Fundamental Research Funds for the Central Universities under Grants ZYGX2018J053 and ZYGX2018J055.

Kang Shin was supported in part by the US National Science Foundation under Grant CNS-1646130.

\section{REFERENCES}

[1] DoD. 2014. United States Department of Defense Suppliers Passive RFID Information Guide, Version 15.0. https://www.acq.osd.mil/ $\log$ /sci/rfid_FAQs.html 